\title{
Lyme Arthritis
}

National Cancer Institute

\section{Source}

National Cancer Institute. Lyme Arthritis. NCI Thesaurus. Code C119026.

Joint inflammation, most often affecting large joints, associated with Lyme disease,

presenting months after infection. 